\title{
MCM3AP-AS1 regulates proliferation, apoptosis, migration, and invasion of breast cancer cells via binding with ZFP36
}

\author{
Tian-Ping Tang^, Chun-Xin Qin, Hao Yu \\ Department of Breast and Thyroid Surgery, Weihai Municipal Hospital, Cheeloo College of Medicine, Shandong University, Weihai, China \\ Contributions: (I) Conception and design: TP Tang; (II) Administrative support: H Yu; (III) Provision of study materials or patients: CX Qin, H Yu; (IV) \\ Collection and assembly of data: All authors; (V) Data analysis and interpretation: CX Qin; (VI) Manuscript writing: All authors; (VII) Final approval \\ of manuscript: All authors. \\ Correspondence to: Dr. Tian-Ping Tang. Department of Breast and Thyroid Surgery, Weihai Municipal Hospital, Heping Road, Huancui Zone, Weihai \\ 264200, China. Email: Lian758@163.com.
}

\begin{abstract}
Background: Previous studies suggest that long noncoding RNA (lncRNA) maintenance complex component 3 associated protein (MCM3AP) antisense RNA 1 (MCM3AP-AS1) has a wide range of functions in several cancers. However, its expression and functions in breast cancer are unclear.

Methods: Reverse transcription polymerase chain reaction (RT-PCR) was used to detect the expression of MCM3AP-AS1. MTT, colony formation and anchorage-independent growth assay were used to examine the effect of MCM3AP-AS1 on growth of breast cancer cells. TUNEL and flow cytometry assay were applied to investigate the role of MCM3AP-AS1 on cell apoptosis. Wound heading and transwell matric penetration assay were used to detect the role of MCM3AP-AS1 on cell motility. RNA pull-down and RIP assay were used to examine the interaction of MCM3AP-AS1 and ZFP36.
\end{abstract}

Results: We found that lncRNA MCM3AP-AS1 was significantly upregulated in breast cancer, which correlated with patients' clinicopathological characteristics. Downregulation of MCM3AP-AS1 substantially inhibited the proliferation, apoptosis, migration, and invasion of breast cancer cells. Further analysis clarified that MCM3AP-AS1 binds with the RNA-binding protein ZFP36 ring finger protein (ZFP36) to regulate the levels of cyclin D1 (CCND1), c-myc (MYC), and matrix metalloproteinase 1 (MMP1).

Conclusions: Our findings show lncRNA MCM3AP-AS1 might be a prognostic factor and a promising therapeutic target for breast cancer therapy.

Keywords: Long noncoding RNA (lncRNA); RNA-binding protein; maintenance complex component 3 associated protein antisense RNA 1 (MCM3AP-AS1); ZFP36 ring finger protein (ZFP36); cell cycle

Submitted Aug 13, 2021. Accepted for publication Oct 20, 2021.

doi: $10.21037 /$ tcr-21-1940

View this article at: https://dx.doi.org/10.21037/tcr-21-1940

\section{Introduction}

In 2018, about 2.1 million new cases of female breast cancer were reported globally, which accounted for almost $25 \%$ of cancer cases among women. Breast cancer is the most commonly occurring cancer in 154 out of 185 countries. It is also the leading cause of cancer death among women in more than 100 countries (1). Therefore, breast cancer is an important health issue worldwide, and there is an urgent need to explore its underlying mechanisms to develop novel therapies to treat patients with breast cancer.

Dysregulation of oncogenes and tumor suppressor genes plays an important role in breast cancer tumorigenesis and progression (2). This occurs in a context in which $70 \%$ of the human genome is identified as encoding noncoding

^ ORCID: 0000-0003-4104-0909. 
RNAs (ncRNAs) $(2,3)$. ncRNAs are classified into two categories, long ncRNAs (lncRNAs) and small ncRNAs, according to their transcript length (4). LncRNAs are larger than $200 \mathrm{nt}$ in length, and research suggests that lncRNAs are involved in many physiological and pathological processes (5), in which they might act as transcriptional modulators, splicing regulators, posttranscriptional processors, enhancers, molecular decoys for miRNAs, chromatin remodelers, or as a guide for protein-protein, protein-DNA, and protein-RNA interactions (6-8). The functions of lncRNAs in cancer attracted our interest, because multiple lncRNAs are dysregulated in cancer. Xue et al. demonstrated that IncRNA HOTAIR is upregulated in tamoxifen-resistant breast cancer tissues, and that upregulation of HOTAIR increased estrogen receptor (ER) levels and its downstream gene regulation to induce tamoxifen resistance (9). Tan et al. showed that lncRNA NORAD is downregulated and is closely correlated with lymph node metastases of and poor prognosis in breast cancer (10). Li et al. found that IncRNA NEAT1 is upregulated in breast cancer and can promote the invasion of breast cancer cells via the microRNA miR211/high mobility group AT-hook 2 (HMGA2) axis (11). Several studies showed that lncRNA maintenance complex component 3 associated protein (MCM3AP) antisense RNA 1 (MCM3AP-AS1) is implicated in the progression of hepatocellular carcinoma (12), glioblastoma (13), papillary thyroid cancer (14), and pancreatic (15), prostate (16), and breast cancer (17). Chen et al. showed MCM3APAS1 is reported to be overexpressed in breast cancer, and MCM3AP/miR-28-5p/CENPF axis accelerates breast cancer progression. However, the authors didn't investigate the expression of MCM3AP-AS1 in breast cancer tissues, and whether MCM3AP is involved in other mechanisms in breast cancer is unclear (17). Previous studies suggest that lncRNA MCM3AP-AS1 has important and wideranging functions in cancer progression. The abovementioned reports showed that MCM3AP-AS1 might play an important role in cancer progression. However, the mechanisms of lncRNA MCM3AP-AS1 in breast cancer tissues remains unclear, and its expression and mechanisms are yet to be clarified.

ZNF36 encodes a member of the Kruppel C2H2type zinc-finger family of proteins. This encoded protein may function as a transcription factor that regulates the expression of GABA type-A receptors. Elevated expression of this gene has been observed in gastric cancer and the encoded protein may stimulate migration and invasion of human gastric cancer cells (18). Silencing of ZNF36 induces apoptosis in human gastric cancer cells (19).

The present study aimed to determine the expression status of lncRNA MCM3AP-AS1 in breast cancer tissues, and its relationship with the clinical characteristics of patients with breast cancer. We also studied the effect of downregulation of MCM3AP-AS1 expression on breast cancer cell viability, migration, and invasion. Moreover, we sought to determine the binding partner(s) of MCM3AP-AS1.

We present the following article in accordance with the MDAR reporting checklist (available at https://dx.doi. org/10.21037/tcr-21-1940).

\section{Methods}

\section{Cells and tissue specimens}

The breast cancer cells ZR-75-30 and MDA-MB-231 were purchased from Shanghai Institutes for Biological Sciences Cell Resource Centre (Shanghai, China), and cultured using Dulbecco's modified Eagle medium (DMEM; ThermoFisher Scientific, Waltham, MA, USA) containing $10 \%$ fetal bovine serum (FBS; Gibco, Grand Island, NY, USA). All cells were cultured in a humidified incubator containing $5 \% \mathrm{CO}_{2}$ at $37^{\circ} \mathrm{C}$.

A total of 30 paired breast cancer and corresponding adjacent normal tissues (ANTs) were collected from the Weihai Municipal Hospital. Before collection, patient consent and approval from the Institutional Research Ethics Committee of Weihai Municipal Hospital were obtained. All procedures performed in this study involving human participants were in accordance with the Declaration of Helsinki (as revised in 2013). None of the patients received any pre-operative therapy. Clinicopathological stage and tumor-node-metastasis (TNM) classifications were evaluated following the criteria stipulated by the American Joint Committee on Cancer (AJCC).

\section{Quantitative real-time polymerase chain reaction (qPCR) and plasmids}

The total RNA was extracted using TRIzol (Invitrogen, Waltham, MA, USA). The nuclear and cytoplasmic fractions were extracted using a Minute ${ }^{\mathrm{TM}}$ Cytoplasmic and Nuclear Extraction Kit (Invent Biotechnologies, Plymouth, MN, USA). qPCR was performed on a 7500 Fast Real Time PCR system (Applied Biosystems, Foster City, CA, USA) using the SYBR Green PCR 
Kit (Invitrogen, Waltham, MA, USA). The primers used were as follows: MCM3AP-AS1, forward, 5'GCTGCTAATGGCAACACTGA-3', reverse, 5' AGGTGCTGTCTGGTGGAGAT-3'; cyclin D1 (CCND1), forward, 5'-TCTACACCGACAACTCCATCCG-3', reverse, 5'- TCTGGCATTTTGGAGAGGAAGTG-3'; c-myc $(M Y C)$, forward, 5'-CCTGGTGCTCCATGAGGAGAC-3', reverse, 5'-CAGACTCTGACCTTTTGCCAGG-3'; matrix metalloproteinase 1 (MMP1), forward, 5'-ATGAAGCAGCCCAGATGTGGAG-3', reverse, 5'-TGGTCCACATCTGCTCTTGGCA-3'.

A lentivector-mediated short-hairpin RNA (shRNA) targeting MCM3AP-AS1 (sh-1 and sh-2) and scrambled plasmids (sh-control) were designed and synthesized by Geneseed Biotech (Guangzhou, China). The process of screening stable cell lines was performed according to previously published methods (12).

\section{Western blotting assay}

Extracted proteins were subjected to western blotting according to previously described methods (20). Equal amounts of protein $(25 \mu \mathrm{g} /$ well) were separated using $10.5 \%$ polyacrylamide gels and transferred onto polyvinylidene fluoride (PVDF) membranes. Subsequently, the membranes were probed with primary antibodies (diluted 1:2,500, Abcam, Cambridge, MA, USA) and then incubated with horseradish peroxidase (HRP)-conjugated secondary antibody (diluted 1:1,500, Abcam, Cambridge, UK). $\beta$-actin was used as the loading control of total protein.

\section{3-(4,5-dimethylthiazol-2-yl)-2,5-diphenyltetrazolium bromide (MTT) assay}

We seeded cells into 96-well plates, put the plates into a cell incubator, and collected the cells at the specified time points. Subsequently, we dyed the cell with $50 \mu \mathrm{L}$ MTT reagent for $4 \mathrm{~h}$. The MTT produced blue crystals formazan. We then dissolved the crystals using $100 \mu \mathrm{L}$ dimethyl sulfoxide (DMSO). We finally detected the cell absorbance using a falcon microplate reader (BD-Labware, San Jose, CA, USA) at $540 \mathrm{~nm}$ wavelength.

\section{Colony formation assay}

We seeded the cells into 12-well plates, and put the plates into a $37^{\circ} \mathrm{C}$ humidified incubator. The cells were cultured for 10 days, and we changed the cell culture every day. When the cell culture was finished, we fixed the cell colonies formed using $4 \%$ paraformaldehyde for $5 \mathrm{~min}$. We then dyed the colonies using $0.1 \%$ crystal violet for $30 \mathrm{~min}$ and finally examined and quantified the colonies with a light microscope.

\section{Anchorage-independent growth assay}

We coated 6-well plates using a mixture including complete cell medium and $1 \%$ agar and placed a mixture of $5 \times 10^{2}$ cells, $2 \mathrm{~mL}$ complete medium, and $0.3 \%$ agar onto the coated plates. Then, the plates were incubated in a $37^{\circ} \mathrm{C}$ humidified incubator for 10 days. Finally, we quantified the colonies larger than $0.1 \mathrm{~mm}$.

\section{Terminal deoxynulceotidyl transferase nick-end-labeling (TUNEL) assay}

We seeded the breast cancer cells on coverslips, and put the coverslips into 24 -well plates. The plates were maintained in a humidified incubator containing $5 \% \mathrm{CO}_{2}$ at $37{ }^{\circ} \mathrm{C}$ for $24 \mathrm{~h}$. Then, sorafenib were used to treat the cells for another $24 \mathrm{~h}$. We subsequently fixed the cells using $4 \%$ paraformaldehyde and used a mixture of $0.1 \%$ sodium citrate and $0.1 \%$ Triton-X-100 to permeabilize the cells. The DNA fragmentation was probed using the TUNEL kit following the manufacturer's instructions (Roche Applied Science, Penzberg, Germany). Finally, we captured the fluorescent images using an EVOS fluorescence microscope (AMG, Bothwell, WA, USA).

\section{Flow cytometry}

We seeded the cells into $10 \mathrm{~cm}$ plates and put the cells into a humidified cell incubator for $72 \mathrm{~h}$. We subsequently collected the cells, washed them twice using phosphatebuffered saline (PBS), and resuspended them with binding buffer. Then, we added a mixture of fluorescein isothiocyanate (FITC) annexin V/RNase A and propidium iodide to the cell plates. The cells were maintained at room temperature in the dark. Thirty min later, the cells were examined using a flow cytometer (Beckman-Coulter, Indianapolis, IN, USA).

\section{Wound bealing assay}

We seeded the cells into 6-well plates and put the plates in a humidified incubator containing $5 \% \mathrm{CO}_{2}$ at $37^{\circ} \mathrm{C}$ until they reached $90 \%$ confluence. Then, we scratched the cell layer 
A

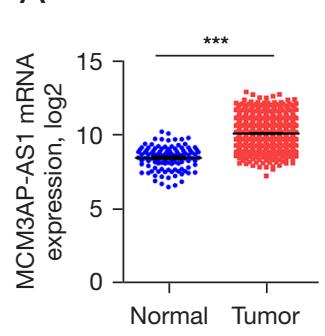

B

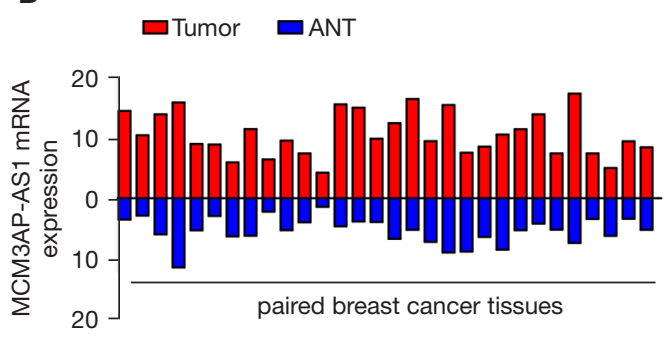

C

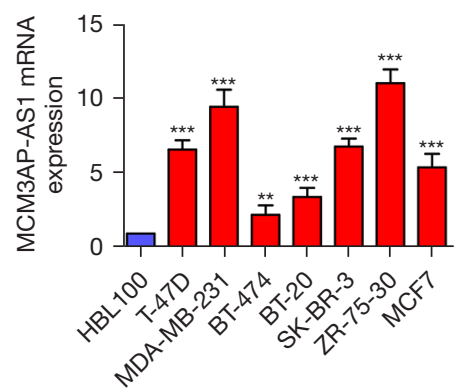

Figure 1 LncRNA MCM3AP-AS1 is significantly increased in breast cancer tissues. (A) The mRNA expression of MCM3AP-AS1 in normal breast tissues and breast cancer tissues using data from the TCGA. (B) The mRNA expression of MCM3AP-AS1 in 30 paired fresh breast cancer tissues. (C) The mRNA expression of MCM3AP-AS1 in in transformed mammary epithelial HBL100 cells, and breast cancer cell lines T-47D, MDA-MB-231, BT-474, BT-20, SK-BR-3, ZR-75-30, and MCF7. **, $\mathrm{P}<0.01$. ***, P<0.001. LncRNA, long noncoding RNA; MCM3AP-AS1, maintenance complex component 3 associated protein antisense RNA 1; mRNA, messenger RNA; TCGA, The Cancer Genome Atlas; ANT, adjacent normal breast tissue.

using a sterile $10 \mu \mathrm{L}$ pipette tip. At 0 and $24 \mathrm{~h}$, we detected the width of the scratch under a microscope (CKX41; Olympus, Tokyo, Japan).

\section{Transwell matrix penetration assay}

We coated the transwell filter chamber with Matrigel (BD Biosciences, Franklin Lakes, NJ, USA) and put the chamber into the 24-well plates after adding cell medium. Then, we seeded $1 \times 10^{4}$ cells into the upper chamber containing cell medium and 10\% FBS (HyClone, Logan, UT, USA). The cells were maintained in a humidified cell incubator for $24 \mathrm{~h}$. We subsequently removed the cells inside the upper chamber with cotton swabs. Then, we used $1 \%$ paraformaldehyde to fix the invaded cells for $10 \mathrm{~min}$ and used hematoxylin to stain the cells for $5 \mathrm{~min}$. Finally, we used a microscope (CKX41; Olympus) to examine and quantify the invaded cells in 10 randomly chosen fields.

\section{RNA pull-down and RNA immunoprecipitation (RIP) assays}

RNA pull-down and RIP assays were performed according to previously described methods (21).

\section{Statistical analysis}

The statistical analysis was performed using SPSS version 20.0 (IBM Corp., Armonk, NY, USA), and the data are presented as means \pm standard deviations. Student's paired $t$-tests were used to analyze the statistical differences between paired tissues, and comparisons among two unpaired groups were analyzed using independent samples $t$-test. The statistical analysis between sh- $1 / 2$ and sh-control were performed using analysis of variance (ANOVA) followed by Dunnett's test. The correlations of lncRNA MCM3AP-AS1 and patients' clinicopathological characteristics were analyzed using the $\chi^{2}$ test. $\mathrm{P}<0.05$ was considered as indicating statistically significant differences. Each experiment was performed three times independently.

\section{Results}

\section{LncRNA MCM3AP-AS1 is upregulated in breast cancer and is closely related to patients' clinical characteristics}

Through analyzing data from the public dataset The Cancer Genome Atlas (TCGA; https://cancergenome.nih.gov/), we found that lncRNA MCM3AP-AS1 was significantly upregulated in breast cancer (Figure 1A). Meanwhile, we determined the expression of MCM3AP-AS1 in 30 fresh paired breast cancer tissues. As demonstrated in Figure $1 B$, the expression of MCM3AP-AS1 was markedly upregulated in breast cancer tissues compared with that in the corresponding ANT. In addition, we investigated the correlation between MCM3AP-AS1 expression and the clinical characteristics of patients with breast cancer. Statistical analysis suggested that the MCM3AP-AS1 expression level was closely associated with clinical stage 
Table 1 Correlation between the clinicopathological features and expression of MCM3AP-AS1

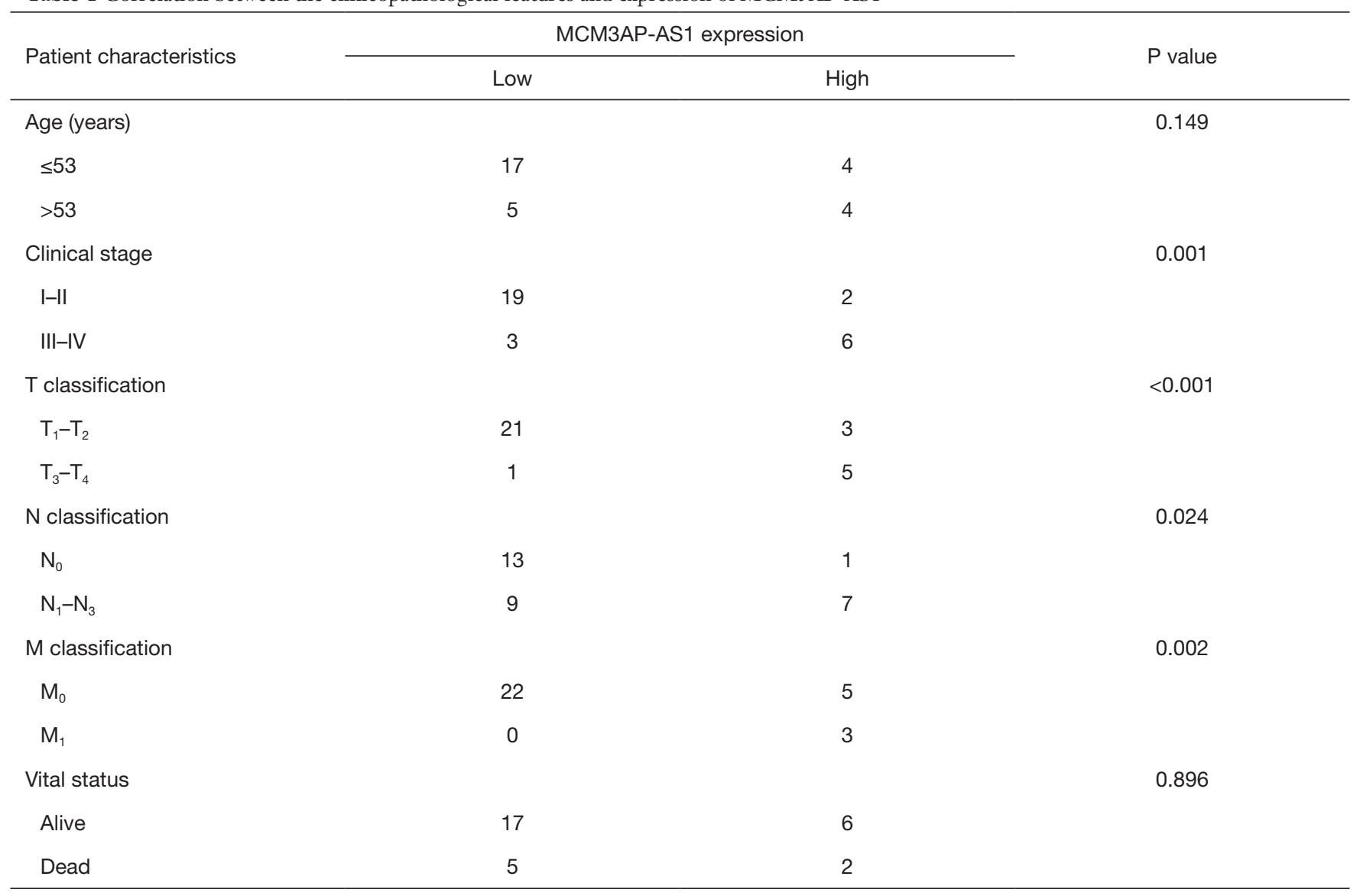

MCM3AP-AS1, maintenance complex component 3 associated protein antisense RNA 1; T, tumor; N, node; M, metastasis.

$(\mathrm{P}=0.001)$, tumor $(\mathrm{T})$ classification $(\mathrm{P}<0.001)$, node $(\mathrm{N})$ classification $(\mathrm{P}=0.024)$, and metastasis $(\mathrm{M})$ classification $(\mathrm{P}=0.002)$ (Table 1). Finally, we detected the expression of MCM3AP-AS1 in transformed mammary epithelial HBL100 cells and breast cancer cell lines (T-47D, MDAMB-231, BT-474, BT-20, SK-BR-3, ZR-75-30 and MCF7) using qPCR. The results showed that the expression of MCM3AP-AS1 in breast cancer cells was upregulated significantly compared with that in the normal transformed mammary epithelial cells (Figure $1 C$ ). The above analysis showed that MCM3AP-AS1 is significantly increased in breast cancer and might be a prognostic factor for poor prognosis of patients with breast cancer.

\section{Downregulation of MCM $A P-A S 1$ inbibits cell growth and induces apoptosis of breast cancer cells}

MCM3AP-AS1 levels were closely correlated with the T classification; therefore, we explored the biological functions of MCM3AP-AS1 related to breast cancer growth. First, MCM3AP-AS1 was stably knocked down in MDA-MB-231 and ZR-75-30 cells using a specific shRNA (Figure 2A). Subsequently, MTT assays showed that downregulation of MCM3AP-AS1 substantially suppressed the proliferation of MDA-MB-231 and ZR-75-30 cells (Figure 2B). Colony formation (Figure 2C) and anchorage-independent growth assays (Figure 2D) also demonstrated that MCM3AP-AS1 knockdown significantly inhibited the growth of MDAMB-231 and ZR-75-30 cells. In addition, TUNEL assays and flow cytometry illustrated that the percentage of apoptotic cells was significantly upregulated in MCM3APAS1-silenced breast cancer cells (Figure 2E-2G). Moreover, knockdown of MCM3AP-AS1 induced cell cycle arrest at the G1 phase in MDA-MB-231 and ZR-75-30 cells (Figure 2H,2I). Taken together, these findings suggest that downregulation of MCM3AP-AS1 significantly inhibits the 
A

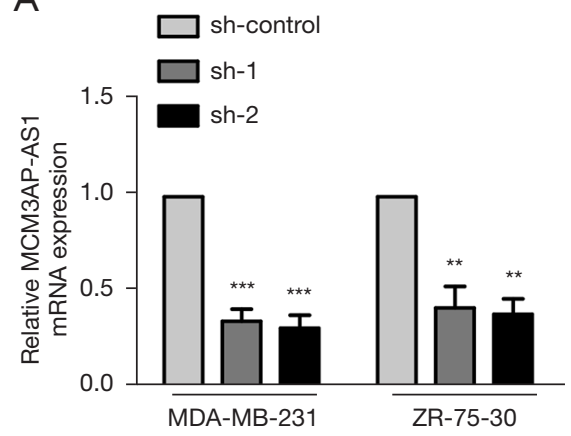

C

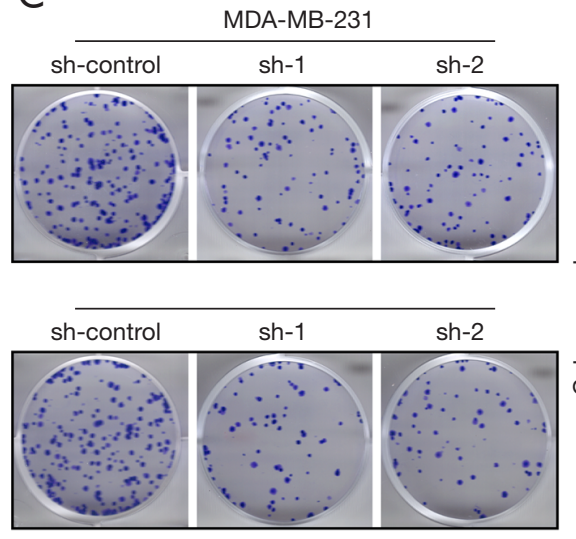

D

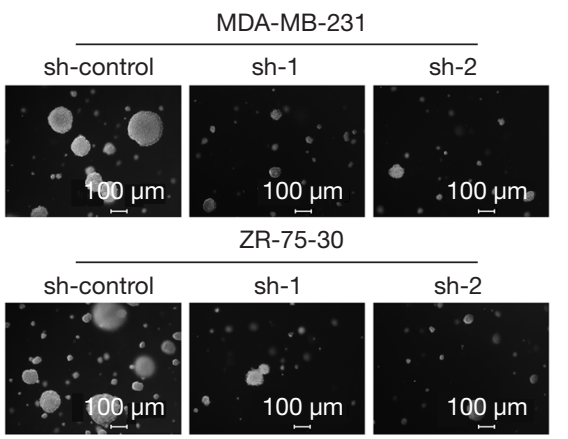

$\mathrm{H}$

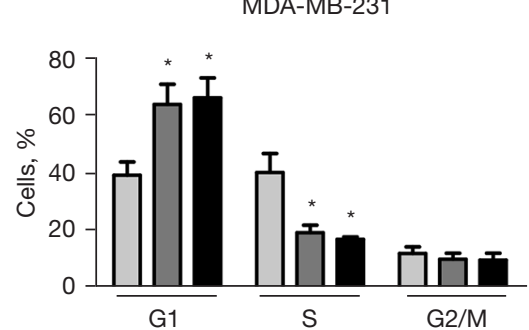

B

$\rightarrow$ sh-contro

MDA-MB-231

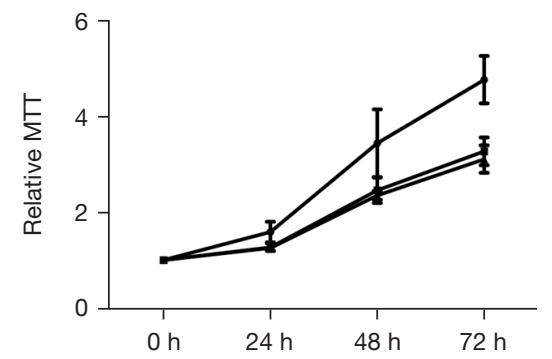

E
ZR-75-30

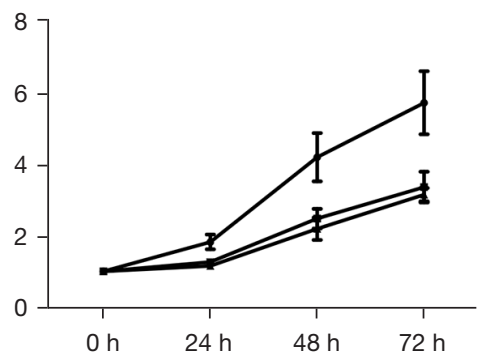

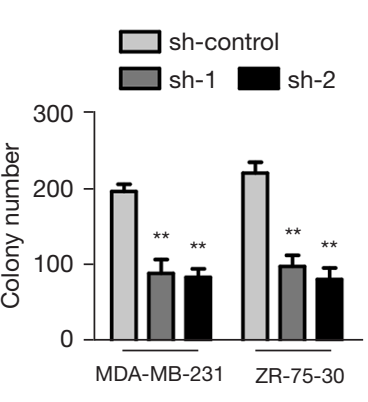
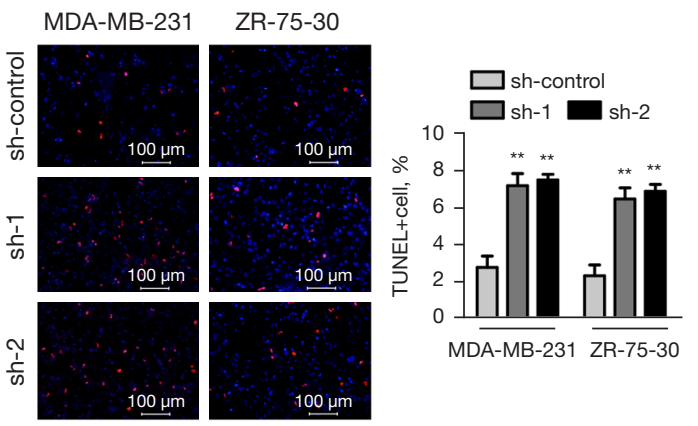

$\mathrm{F}$
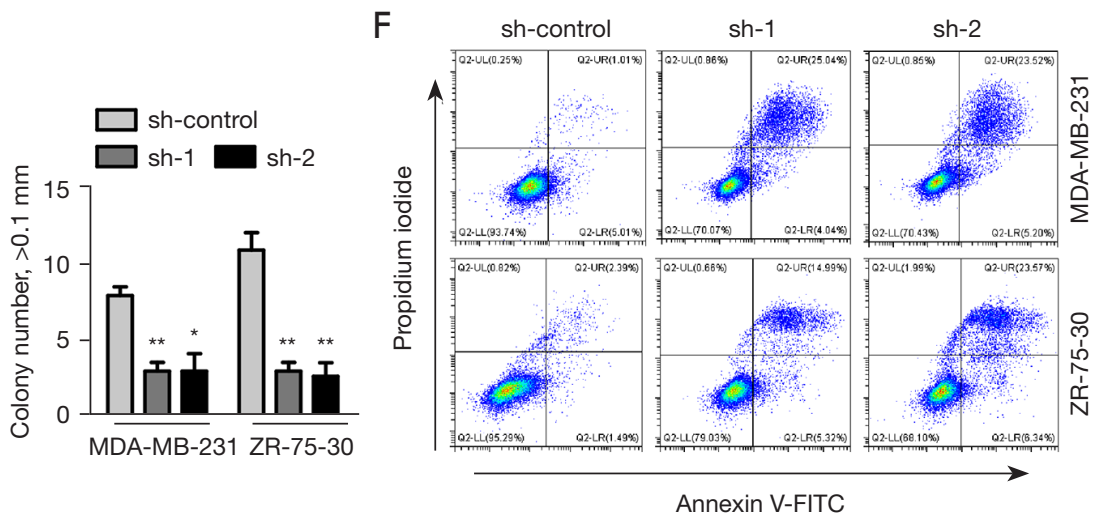

G

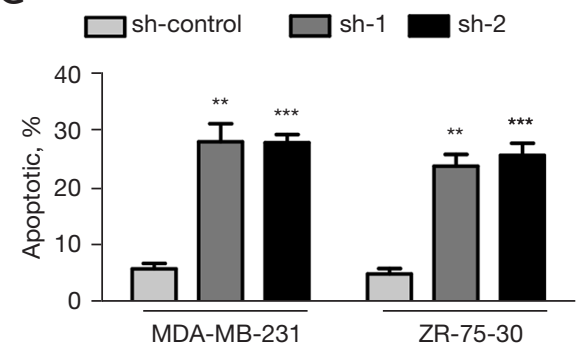



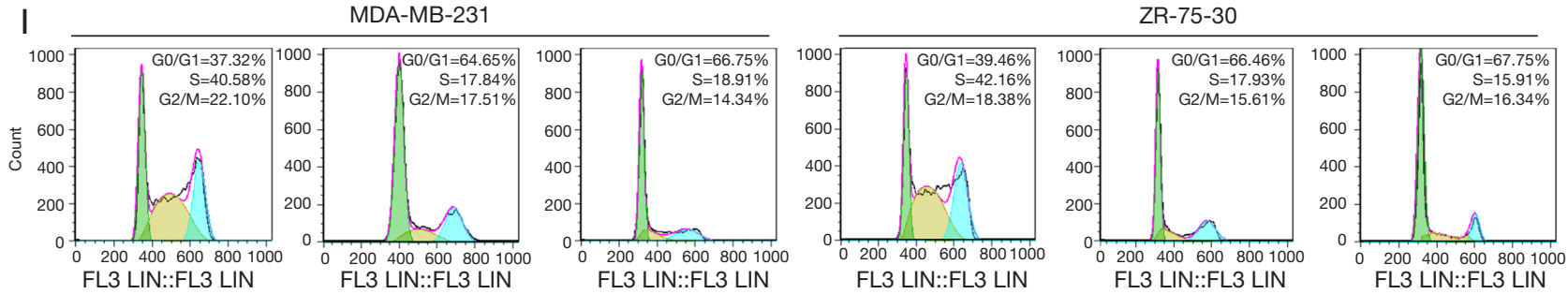

Figure 2 Downregulation of MCM3AP-AS1 inhibits cell growth and induces apoptosis of breast cancer cells. (A) Relative expression of MCM3AP-AS1 in the indicated stable cell lines. (B) Downregulation of MCM3AP-AS1 substantially inhibits the proliferation of MDAMB-231 (left panel) and ZR-75-30 (right panel) using the MTT assay. (C) Representative images (left panel) and quantitative analysis (right panel) of the indicated cell lines using colony formation assays. (D) Representative images (left panel) and quantitative analysis (right panel) of the indicated cell lines using anchorage-independent growth assays. (E) Downregulation of MCM3AP-AS1 increases the number of TUNEL \pm cells compared with the scramble group under the treatment with sorafenib. (F) Representative image of apoptotic cells from a flow cytometry assay. $(\mathrm{G})$ Apoptotic cells in different cell lines. $(\mathrm{H})$ Flow cytometry assay showing that downregulation of MCM3APAS1 results in cell cycle G1 phase arrest in MDA-MB-231 (left panel) and ZR-75-30 (right panel) cells. (I) The representative image of cell cycle. *, $\mathrm{P}<0.05$; **, $\mathrm{P}<0.01$; ***, $\mathrm{P}<0.001$. MCM3AP-AS1, maintenance complex component 3 associated protein antisense RNA 1; MTT, 3-(4,5-dimethylthiazol-2-yl)-2,5-diphenyltetrazolium bromide; TUNEL, terminal deoxynulceotidyl transferase nick-end-labeling.

proliferation and cell cycle transition of breast cancer cells, leading to their apoptosis.

\section{Downregulation of MCM $3 A P-A S 1$ suppresses the migration and invasion of breast cancer cells}

MCM3AP-AS1 levels were related to the $\mathrm{N}$ and $\mathrm{M}$ classifications; therefore, we further checked the biological roles of MCM3AP-AS1 in migration and invasion of breast cancer. As shown in Figure 3A,3B, downregulation of MCM3AP-AS1 significantly reduced the migration and invasion of breast cancer cells, as assessed using wound healing and transwell assays, respectively. Thus, knockdown of MCM3AP-AS1 significantly inhibited the migration and invasion of breast cancer cells.

\section{MCM3AP-AS1 mainly located in the nucleus}

The location of RNA is regarded as an important clue to its function (22). Therefore, we used the long noncoding (lnc) ATLAS website (http://lncatlas.crg.eu/) to analyze the subcellular location of MCM3AP-AS1. As shown in Figure 4A, MCM3AP-AS1 was mainly located in the nucleus, which was confirmed using cell lines. We performed a cytoplasmic and nuclear extraction experiment, and then detected the level of MCM3AP-AS1 in the cytoplasm and nucleus. The results showed that MCM3AP-AS1 was mainly located in the nucleus (Figure 4B).

\section{MCM $3 A P-A S 1$ can directly bind with RNA-binding protein ZFP36 ring finger protein (ZFP36)}

To investigate the molecular mechanisms of MCM3APAS1's effects on breast cancer progression, we predicted its binding protein through analyzing a public dataset, the database of RNA-Binding Protein Specificities (RBPDB; http://rbpdb.ccbr.utoronto.ca/index.php). We found that the binding protein that has the highest score is RNAbinding protein ZFP36. Next, we verified the prediction using molecular experiments. An RNA pull-down assay illustrated that ZFP36 was present in the complexes pulled down using biotinylated MCM3AP-AS1 but was not present in the complexes pulled down using immunoglobulin $\mathrm{G}$ (IgG) (Figure 4C). Correspondingly, an RIP assay also demonstrated that MCM3AP-AS1 was enriched in the lysate pulled down using the antibody recognizing ZFP36, but not in the lysate pulled down using IgG (Figure 4D). The RNA pull-down and RIP assays suggested that lncRNA MCM3AP-AS1 can bind with ZNF36. ZNF36 is a member of a small family of tandem Cys3His zinc finger proteins and promotes rapid decay of adenylate-uridylate-rich elements (ARE)-containing messenger RNA (mRNA) $(23,24)$. ZNF36 can regulate several oncogenes and growth factors such as CCND1, MMP1, and MYC $(25,26)$. Therefore, we detected the mRNA of CCND1, MYC, and $M M P 1$. As shown in Figure $4 E, 4 F$, the mRNA levels of $C C N D 1, M Y C$, and $M M P 1$ decreased significantly in MCM3AP-AS1-silenced cells. Taken together, these results 

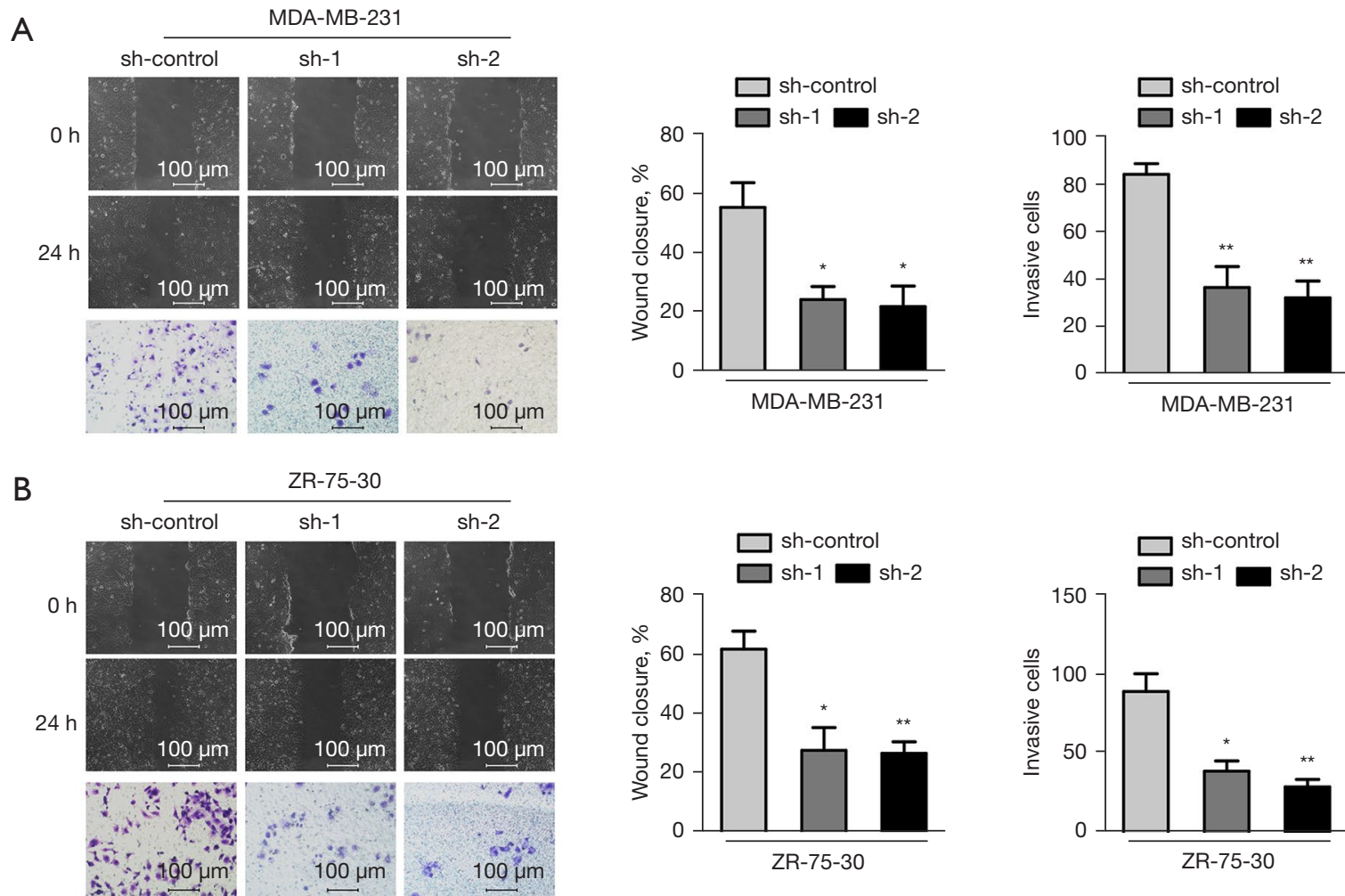

Figure 3 Downregulation of MCM3AP-AS1 suppresses the migration and invasion of breast cancer cells. (A) Representative image (left panel) and quantitative analysis (right panel) of wound healing and transwell assays stained by hematoxylin using MDA-MB-231 cells, respectively. (B) Representative image (left panel) and quantitative analysis (right panel) of wound healing and transwell assays stained by hematoxylin using ZR-75-30 cells, respectively. *, $\mathrm{P}<0.05$; **, $\mathrm{P}<0.001$. MCM3AP-AS1, maintenance complex component 3 associated protein antisense RNA 1.

show that lncRNA MCM3AP-AS1 can bind with RNAbinding protein ZFP36 to regulate the expressions of CCND1, MYC, and MMP1.

\section{Discussion}

To date, multiple lncRNAs have been discovered using functional genomics research. LncRNAs interact with DNA, RNA, or proteins to play crucial functions in many biological processes and diseases at the transcriptional or post-transcriptional level (27). Therefore, it is important to explore potential lncRNAs that might serve as prognostic factors or therapeutic targets. Consequently, we combined data from previous studies and a publicly available dataset and found that lncRNA MCM3AP-AS1 plays an important and wide-ranging role in the progression of multiple cancers. The results of the present study showed that lncRNA MCM3AP-AS1 expression is increased significantly in breast cancer tissues. Further correlation analysis of clinicopathological characteristics and MCM3AP-AS1 showed that MCM3AP-AS1 is closely related with clinical stage and TNM classification, suggesting that MCM3APAS1 might be a prognostic factor for poor prognosis of patients with breast cancer. Chen et al. showed that MCM3AP-AS1 is upregulated in breast cancer cells and can regulate CENPF expression (17). However, their studies did not include correlation analysis of MCM3AP-AS1 with clinical features in breast cancer tissues. Our study extended our understanding of the importance of MCM3AP-AS1 in breast cancer. However, the number of specimens analyzed in the present study was low; therefore, we will increase the number of specimens in our future study.

LncRNAs can function as oncogenes or tumor suppressors. Alterations of lncRNAs promote tumorigenesis and metastasis (28). The widely studied LncRNA HOTAIR is involved in breast cancer progression, such 
A

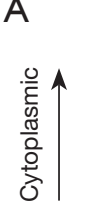
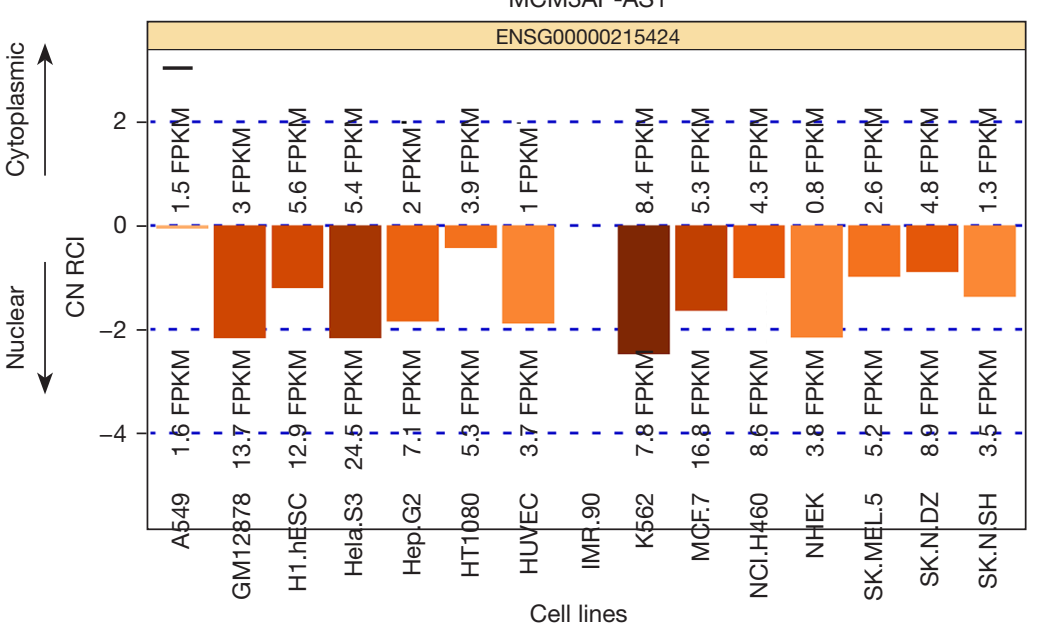

Nuclear expression, log10 (FPKM) $\square 0.4 \square 0.8$
MCM3AP-AS1

ENSG00000215424
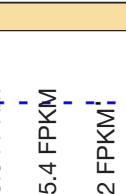

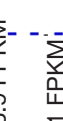

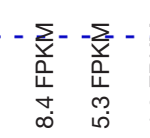
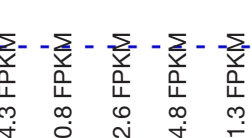

C

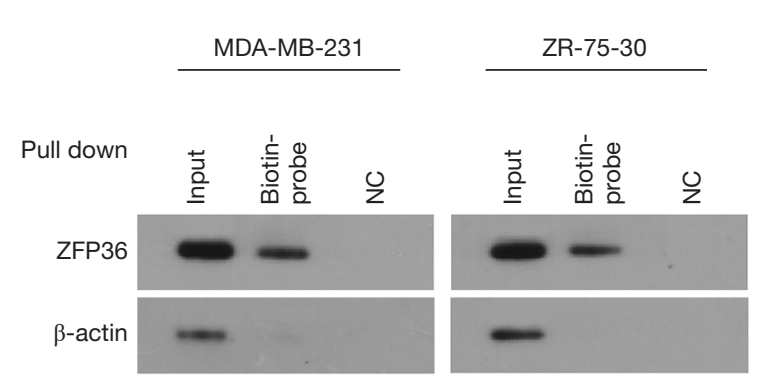

$E$

MDA-MB-231

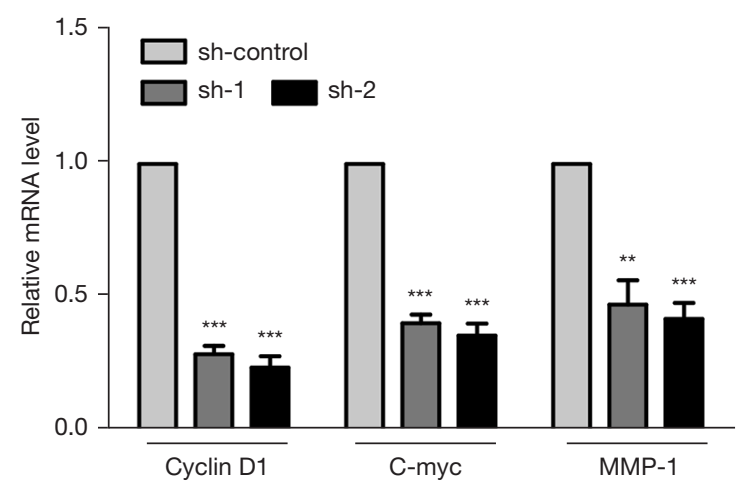

B

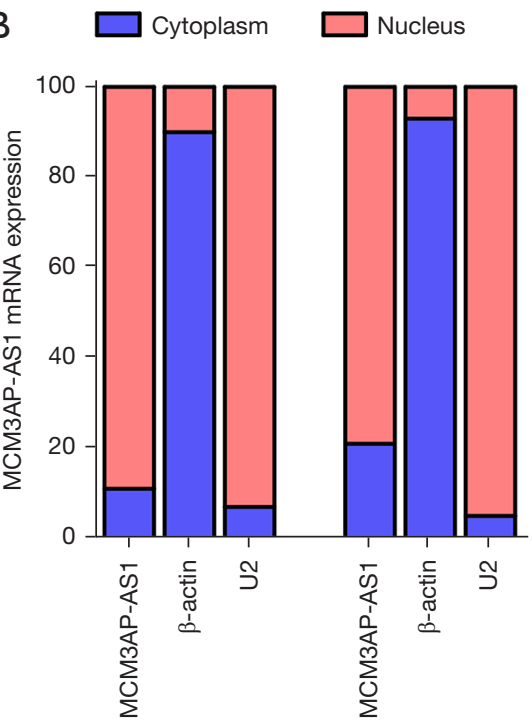

D

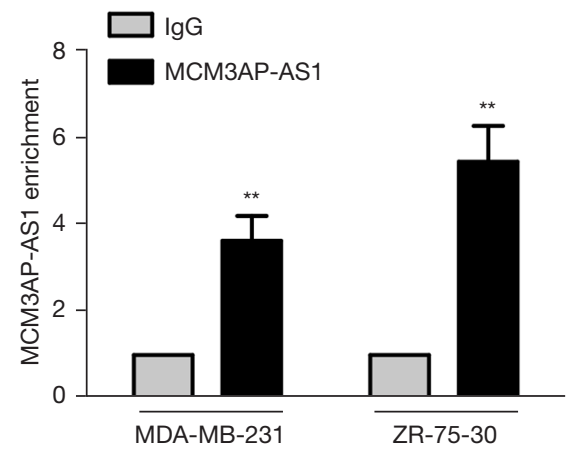

$\mathrm{F}$

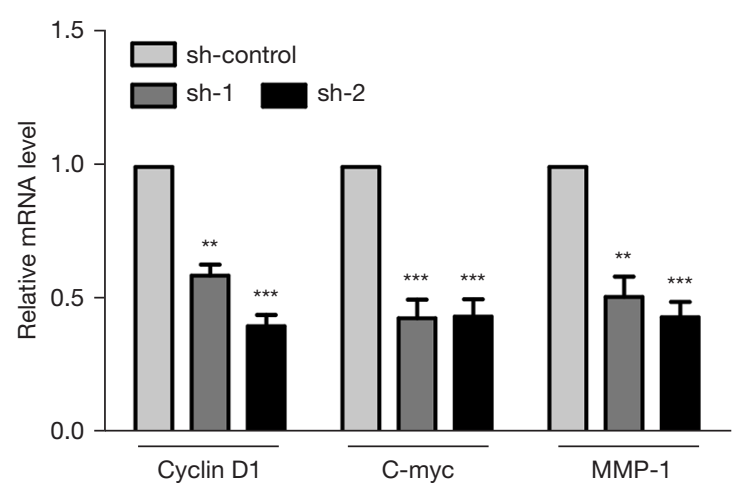

Figure 4 MCM3AP-AS1 can directly bind with the RNA-binding protein ZFP36. (A) Analysis of MCM3AP-AS1 subcellular location using the lnc ATLAS website. (B) MCM3AP-AS1 levels in the nucleus and cytoplasm. (C) An RNA pull-down assay showing that ZFP36 can bind directly with MCM3AP-AS1. (D) An RIP assay showing that MCM3AP-AS1 is enriched in the lysate pulled down by an antibody recognizing ZFP36. (E) mRNA levels of CCND1, MYC, and MMP1 are significantly decreased in MCM3AP-AS1-silenced cells as assessed using qPCR assay using MDA-MD-231 cells. (F) mRNA levels of CCND1, MYC, and MMP1 are significantly decreased in MCM3AP-AS1-silenced cells as assessed using qPCR assay using ZR-75-30 cells. ${ }^{* *}, \mathrm{P}<0.01$; ${ }^{* * *}, \mathrm{P}<0.001$. MCM3AP-AS1, maintenance complex component 3 associated protein antisense RNA 1; ZFP36, ZFP36 ring finger protein; lnc, long noncoding; mRNA, messenger RNA; CCND1, cyclin D1; MYC, c-myc; $M M P 1$, matrix metalloproteinase 1; qPCR, quantitative real-time polymerase chain reaction; RIP, RNA immunoprecipitation. 
as via epithelial-to-mesenchymal transition (EMT) (29), metastasis (30), and tamoxifen resistance (9). LncRNA SNAR also regulates proliferation, migration, and invasion in breast cancer $(31,32)$. Our study showed that lncRNA MCM3AP-AS1 is involved in breast cancer progression. Downregulation of MCM3AP-AS1 significantly inhibited the proliferation, migration, invasion, and cell cycle transition, leading to breast cancer cell apoptosis. However, the in vivo functions of MCM3AP-AS1 in breast cancer require further clarification in future studies.

Most lncRNAs exert their biological role in association with RNA-binding proteins, which can interact with multiple different RNAs (33). Corresponding, our study showed that MCM3AP-AS1 can bind with ZNF36 through RNA pull-down and RIP assays in breast cancer cells. It has been reported that $\mathrm{ZNF} 36$ can function as a transcription factor, and can interact with AREs through tandem $\mathrm{CCCH}$ zinc fingers in the initiation and development of cancers through modulating mRNA decay $(34,35)$. Our study showed that ZFP36 can further regulate the expression of oncogenes such as CCND1, MYC, and MMP1 on transcription level. Our findings showed that lncRNA MCM3AP-AS1 might be a promising therapeutic target in breast cancer. However, we aim to investigate other mechanisms of lncRNA MCM3AP-AS1 in breast cancer progression in our future research.

\section{Acknowledgments}

Funding: None.

\section{Footnote}

Reporting Checklist: The authors have completed the MDAR reporting checklist. Available at https://dx.doi. org/10.21037/tcr-21-1940

Data Sharing Statement: Available at https://dx.doi. org/10.21037/tcr-21-1940

Conflicts of Interest: All authors have completed the ICMJE uniform disclosure form (available at https://dx.doi. org/10.21037/tcr-21-1940). The authors have no conflicts of interest to declare.

Ethical Statement: The authors are accountable for all aspects of the work in ensuring that questions related to the accuracy or integrity of any part of the work are appropriately investigated and resolved. All procedures performed in this study involving human participants were in accordance with the Declaration of Helsinki (as revised in 2013). The study was approved by the Institutional Research Ethics Committee of Weihai Municipal Hospital and informed consent was taken from all the patients.

Open Access Statement: This is an Open Access article distributed in accordance with the Creative Commons Attribution-NonCommercial-NoDerivs 4.0 International License (CC BY-NC-ND 4.0), which permits the noncommercial replication and distribution of the article with the strict proviso that no changes or edits are made and the original work is properly cited (including links to both the formal publication through the relevant DOI and the license). See: https://creativecommons.org/licenses/by-nc-nd/4.0/.

\section{References}

1. Bray F, Ferlay J, Soerjomataram I, et al. Global cancer statistics 2018: GLOBOCAN estimates of incidence and mortality worldwide for 36 cancers in 185 countries. CA Cancer J Clin 2018;68:394-424.

2. Hemminki K, Hemminki A, Försti A, et al. Genetics of gallbladder cancer. Lancet Oncol 2017;18:e296.

3. Yan X, Hu Z, Feng Y, et al. Comprehensive genomic characterization of long non-coding RNAs across human cancers. Cancer Cell 2015;28:529-40.

4. Batista PJ, Chang HY. Long noncoding RNAs: cellular address codes in development and disease. Cell 2013;152:1298-307.

5. Schmitt AM, Chang HY. Long noncoding RNAs in cancer pathways. Cancer Cell 2016;29:452-63.

6. Mercer TR, Dinger ME, Mattick JS. Long non-coding RNAs: insights into functions. Nat Rev Genet 2009;10:155-9.

7. Wang KC, Chang HY. Molecular mechanisms of long noncoding RNAs. Mol Cell 2011;43:904-14.

8. Chandra Gupta S, Nandan Tripathi Y. Potential of long non-coding RNAs in cancer patients: from biomarkers to therapeutic targets. Int J Cancer 2017;140:1955-67.

9. Xue X, Yang YA, Zhang A, et al. LncRNA HOTAIR enhances ER signaling and confers tamoxifen resistance in breast cancer. Oncogene 2016;35:2746-55.

10. Tan BS, Yang MC, Singh S, et al. LncRNA NORAD is repressed by the YAP pathway and suppresses lung and breast cancer metastasis by sequestering S100P. Oncogene 2019;38:5612-26.

11. Li X, Wang S, Li Z, et al. The lncRNA NEAT1 facilitates 
cell growth and invasion via the miR-211/HMGA2 axis in breast cancer. Int J Biol Macromol 2017;105:346-53.

12. Wang Y, Yang L, Chen T, et al. A novel lncRNA MCM3AP-AS1 promotes the growth of hepatocellular carcinoma by targeting miR-194-5p/FOXA1 axis. Mol Cancer 2019;18:28.

13. Yang C, Zheng J, Xue Y, et al. The effect of MCM3APAS1/miR-211/KLF5/AGGF1 axis regulating glioblastoma angiogenesis. Front Mol Neurosci 2018;10:437.

14. Liang M, Jia J, Chen L, et al. LncRNA MCM3AP-AS1 promotes proliferation and invasion through regulating miR-211-5p/SPARC axis in papillary thyroid cancer. Endocrine 2019;65:318-26.

15. Yang M, Sun S, Guo Y, et al. Long non-coding RNA MCM3AP-AS1 promotes growth and migration through modulating FOXK1 by sponging miR-138-5p in pancreatic cancer. Mol Med 2019;25:55.

16. Li X, Lv J, Liu S. MCM3AP-AS1 KD inhibits proliferation, invasion, and migration of PCa cells via DNMT1/DNMT3 (A/B) methylation-mediated upregulation of NPY1R. Mol Ther Nucleic Acids 2020;21:264-5.

17. Chen Q, Xu H, Zhu J, et al. LncRNA MCM3AP-AS1 promotes breast cancer progression via modulating miR-28-5p/CENPF axis. Biomed Pharmacother 2020;128:110289.

18. Li Y, Tan BB, Zhao Q, et al. ZNF139 promotes tumor metastasis by increasing migration and invasion in human gastric cancer cells. Neoplasma 2014;61:291-8.

19. Fan L, Tan B, Li Y, et al. Silencing of ZNF139-siRNA induces apoptosis in human gastric cancer cell line BGC823. Int J Clin Exp Pathol 2015;8:12428-36.

20. Yang H, Cho ME, Li TW, et al. MicroRNAs regulate methionine adenosyltransferase 1A expression in hepatocellular carcinoma. J Clin Invest 2013;123:285-98.

21. Zhang J, Li Z, Liu L, et al. Long noncoding RNA TSLNC8 is a tumor suppressor that inactivates the interleukin-6/STAT3 signaling pathway. Hepatology 2018;67:171-87.

22. St Laurent G, Wahlestedt C, Kapranov P. The Landscape of long noncoding RNA classification. Trends Genet 2015;31:239-51.

23. Lai WS, Carballo E, Thorn JM, et al. Interactions of

Cite this article as: Tang TP, Qin CX, Yu H. MCM3AP-AS1 regulates proliferation, apoptosis, migration, and invasion of breast cancer cells via binding with ZFP36. Transl Cancer Res 2021;10(10):4478-4488. doi: 10.21037/tcr-21-1940
$\mathrm{CCCH}$ zinc finger proteins with mRNA. Binding of tristetraprolin-related zinc finger proteins to Au-rich elements and destabilization of mRNA. J Biol Chem 2000;275:17827-37.

24. Sanduja S, Blanco FF, Dixon DA. The roles of TTP and BRF proteins in regulated mRNA decay. Wiley Interdiscip Rev RNA 2011;2:42-57.

25. Marderosian M, Sharma A, Funk AP, et al. Tristetraprolin regulates Cyclin D1 and c-Myc mRNA stability in response to rapamycin in an Akt-dependent manner via p38 MAPK signaling. Oncogene 2006;25:6277-90.

26. Upadhyay R, Sanduja S, Kaza V, et al. Genetic polymorphisms in RNA binding proteins contribute to breast cancer survival. Int J Cancer 2013;132:E128-38.

27. Peng WX, Koirala P, Mo YY. LncRNA-mediated regulation of cell signaling in cancer. Oncogene 2017;36:5661-7.

28. Bhan A, Soleimani M, Mandal SS. Long noncoding RNA and cancer: a new paradigm. Cancer Res 2017;77:3965-81.

29. Pawłowska E, Szczepanska J, Blasiak J. The long noncoding RNA HOTAIR in breast cancer: does autophagy play a role? Int J Mol Sci 2017;18:2317.

30. Gupta RA, Shah N, Wang KC, et al. Long non-coding RNA HOTAIR reprograms chromatin state to promote cancer metastasis. Nature 2010;464:1071-6.

31. Lee J, Jung JH, Chae YS, et al. Long noncoding RNA snaR regulates proliferation, migration and invasion of triple-negative breast cancer cells. Anticancer Res 2016;36:6289-95.

32. Xu ST, Xu JH, Zheng ZR, et al. Long non-coding RNA ANRIL promotes carcinogenesis via sponging miR-199a in triple-negative breast cancer. Biomed Pharmacother 2017;96:14-21.

33. Ferrè F, Colantoni A, Helmer-Citterich M. Revealing protein-lncRNA interaction. Brief Bioinform 2016;17:106-16.

34. Mukherjee N, Jacobs NC, Hafner M, et al. Global target mRNA specification and regulation by the RNA-binding protein ZFP36. Genome Biol 2014;15:R12.

35. Gupta G, Bebawy M, Pinto TJA, et al. Role of the Tristetraprolin (Zinc Finger Protein 36 Homolog) Gene in Cancer. Crit Rev Eukaryot Gene Expr 2018;28:217-21. 